\title{
PDGF-AA and BDNF Promote Neural Stem Cell Differentiation
}

John Soltys, Christopher Perrone, Julia Knight, Yang Mao-Draayer*

Neurology Department, University of Vermont College of Medicine, Burlington VT, 05405, USA

\begin{abstract}
Neural progenitor cells (NPCs) are capable of differentiating into oligodendrocytes, cells capable of remyelinating MS lesions. Brain-derived neurotrophic factor (BDNF) and platelet-derived growth factor AA (PDGF-AA) are two neurotrophic factors that may stimulate NPC differentiation and likely play a role in MS pathogenesis. While several studies have demonstrated the proliferative and protective capabilities of these factors, an understanding of their direct actions on NPCs is required in order to better understand how these factors can be used therapeutically to promote remyelination. In this study, we investigate how BDNF and PDGF-AA influence postnatal mNPC physiology. We identify that neither factor promotes NPC proliferation or protects against apoptosis, and that both factors promote NPC differentiation into the neuronal and oligo-lineage in a time-dependent fashion. PDGF-AA specifically induces oligo-lineage differentiation. These findings highlight the importance of neurotrophic factors in stimulating NPC differentiation, and offer insight into how to develop neurotrophic factor replacement therapies to achieve the goal of remyelination.
\end{abstract}

Keywords: Neural stem cell; PDGF-AA; BDNF; Oligodendrocyte differentiation; Multiple sclerosis

Abbreviations: NPCs: Neural Progenitor Cells; BDNF: BrainDerived Neurotrophic Factor; PDGF-AA: Platelet-Derived Growth Factor AA; MS: Multiple Sclerosis; CNS: Central Nervous System; OPCs: Oligoprogenitor Cells

\section{Introduction}

Multiple sclerosis (MS) manifests as inflammatory, demyelinating central nervous system (CNS) lesions. The harsh lesion microenvironment does not support the successful remyelination of damaged axons. Acute lesions that are capable of limited remyelination ultimately progress into chronic lesions that are incapable of remyelination altogether $[1,2]$. This failure has been attributed to a depletion of oligoprogenitor cells (OPCs), cells capable of generating new oligodendrocytes [3,4]. While current MS therapies succeed in slowing disease progression, no therapy specifically targets this process of nerve regeneration. Neural stem/progenitor cell (NPC) therapies may be able to fill this gap as they are capable of differentiating into mature oligodendrocytes [5]. However, our limited understanding of the growth factors required for NPC differentiation has slowed the development of these therapies.

The observation that neurotrophic factor expression is required for CNS development highlights the potential role that this peptide family plays on NPC physiology [6,7]. Brain-derived neurotrophic factor (BDNF) and platelet-derived growth factor AA (PDGF) are two such factors that are required for the development of CNS connections and have also been implicated in MS progression. For example, BDNF levels positively correlate with response to interferon-beta treatment $[8,9]$ and macrophages - cells present in the lesion microenvironment - release PDGF-AA in response to inflammatory stimuli [10]. Furthermore, PDGF-AA levels in the CSF decrease with disease progression [11], and large numbers of cells expressing the PDGF receptor a (PDGFRa) are present in MS lesions [12,13]. Recent studies have also highlighted the potential that these factors have in promoting remyelination in vivo. BDNF promotes repair/remyelination in a model of spinal cord transection [14] as well as experimental autoimmune encephalitis (EAE) $[15,16]$ and PDGF injection produces a better remyelination of inflammatory lesions [17]. Thus, identifying the mechanisms by which these two factors work may lead to insight into how to better manipulate the MS microenvironment to promote remyelination.

Many of the investigations looking into the mechanism behind these factors have been done as described in vivo, as well as embryonic NPCs $[18,19]$ or OPCs [20] in vitro. The observation that NPCs are present at high levels in active, inflammatory MS lesions raises the question as to how BDNF and PDGF specifically influence post-natal NPC physiology as these cells form the population that differentiates into OPCs and ultimately mature oligodendrocytes [21]. Therefore, this study investigates the interactions of BDNF and PDGF on NPC physiology. We demonstrate that BDNF and PDGF-AA do not induce NPC proliferation or protect against apoptosis. Both factors induce NPC differentiation into the neuronal and oligodendrocyte lineages in a time dependent manner.

\section{Materials and Methods}

Isolation of murine neural progenitor cells (mNPCs) and cell culture

Dr. Jeffrey Spees at the University of Vermont College of Medicine Stem Cell Core Facility isolated mNPC's from postnatal day four C57/ BL6 mouse brains as previously described [22]. Progenitor cells and neurospheres were cultured in the following "complete medium:" Neurobasal-A media (Invitrogen, Carlsbad, CA) with B27; $10 \mathrm{ng} / \mathrm{mL}$ epidermal growth factor (EGF); $10 \mathrm{ng} / \mathrm{mL}$ basic fibroblast growth factor (FGF); $2 \mathrm{mM}$ L-glutamine, 100 units/mL penicillin; $100 \mu \mathrm{g} / \mathrm{mL}$ streptomycin. NPCs (passages 2, 4 and 5) were cultured on poly D-lysine and laminin coated plates. For all experiments, NPCs were grown to 70-

*Corresponding author: Yang Mao-Draayer, Neurology Department, UVM College of Medicine, Burlington VT, USA, Tel: 802-656-2093; Fax: 802-6564674; E-mail: Draayer@vtmednet.org

Received October 09, 2011; Accepted November 01, 2011; Published November 18,2011

Citation: Soltys J, Perrone C, Knight J, Mao-Draayer Y (2011) PDGF-AA and BDNF Promote Neural Stem Cell Differentiation. J Neurol Neurophysiol S4. doi:10.4172/2155-9562.S4-002

Copyright: ( 2011 Soltys J, et al. This is an open-access article distributed under the terms of the Creative Commons Attribution License, which permits unrestricted use, distribution, and reproduction in any medium, provided the original author and source are credited. 

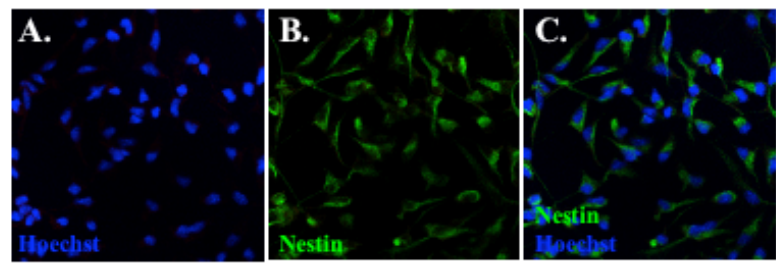

Figure 1: Demonstration of a pure NPC population. Immunohistochemistry was used to verify that we have a pure NPC population. Stains against the neura stem cell marker nestin and the nuclear marker hoechst demonstrate that we have a pure NPC population as every cell (denoted by Hoechst) co-expresses nestin.

$80 \%$ confluency in complete media (CM, described above), then rinsed with EBSS to remove any growth factors, and then treatments were added. Treatments (PDGF-AA or BDNF) were performed in minimal media (MM), which is CM deprived of growth factors EGF and FGF. EGF and FGF stimulate stem cell proliferation to maintain NPCs in the proliferative, non-differentiated state; thus removing them from the media allows us to determine the influence that the specific treatment has on NPC proliferation/differentiation.

\section{Reagents}

PDGF-AA (Peprotech) was reconstituted in.1\% BSA and treatments occurred at $10 \mathrm{ng} / \mathrm{ml}$ or $50 \mathrm{ng} / \mathrm{ml}$. BDNF (R\&D) was reconstituted in $.1 \% \mathrm{BSA}$ and treatments occurred at $10 \mathrm{ng} / \mathrm{ml}$ or $100 \mathrm{ng} / \mathrm{ml}$.
Cell proliferation: mNPC growth curve: $50-100,000$ cells were plated onto each well of a 6 well-plate. Every 24 hours, one well would be counted. Cells were trypsonized from the well and counted manually using a standard cell counter under a microscope.

Bromodeoxyuridine (BrdU; BD Pharmingen) and Ki67 Assays: BrdU is an $S$ phase cell cycle marker which gets incorporated into dividing cells and Ki67 is an antigen expressed during all phases of the cell cycle. The BrdU assay was performed per manufacturer instructions. Briefly, cells in monolayer were first pulsed with $10 \mu \mathrm{M}$ BrdU overnight (16hrs). Cells $\left(1 \times 10^{6}\right.$ per $\left.50 \mu \mathrm{L}\right)$ were permeabilized with BD Cytofix/ Cytoperm $^{\mathrm{TM}}$ Buffer followed by BD Cytoperm Plus Buffer ${ }^{\mathrm{TM}}$. After fixation/ permeabilization process, cells were treated with DNase to expose incorporated BrdU. To detect proliferating cells, samples were then incubated with FITC-conjugated anti-BrdU antibody for $20 \mathrm{~min}$ and rinsed before analyzing on the flow cytometer. Incubations and rinses were performed with either DPBS $(+3 \%$ FCS $/ 0.09 \%$ sodium azide) or $\mathrm{BD}$ Perm/Wash ${ }^{\mathrm{TM}}$ Buffer. For the Ki67 assay, cells were fixed as per the BrdU assay and a monoclonal antibody against Ki67 (BD Pharmingen) was applied for 30 minutes at room temperature, washed in PBS washing buffer (1\%FBS, $\left.0.09 \% \mathrm{NaN}_{3}, \mathrm{pH} 7.2\right)$, and Alexafluor488-conj (BD Via Probe) was added as the secondary antibody used for detection. All flow cytometry was performed on a BD LSRII flow cytometer, and data analyzed with Flow Jo software. Proliferation experiments were performed on day 4 of all treatments. All data is presented as the percentage of cells staining positive for dye incorporation over the total number of nuclei counted.

\section{A. mNPC Growth Curve}
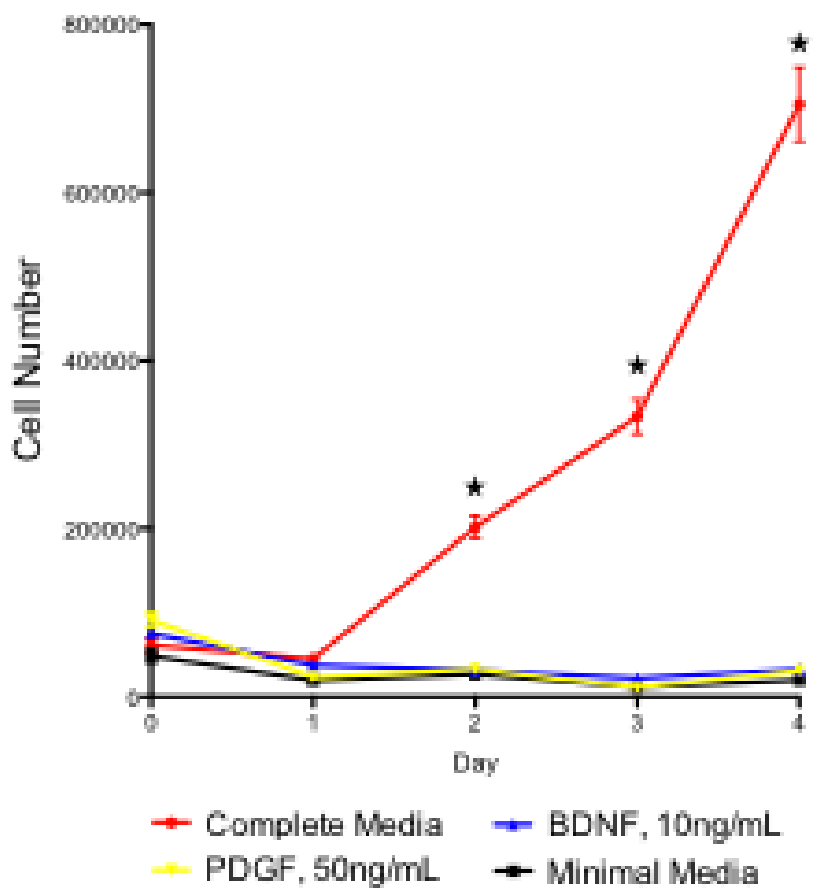

\section{B. Ki67 Proliferation Assay}

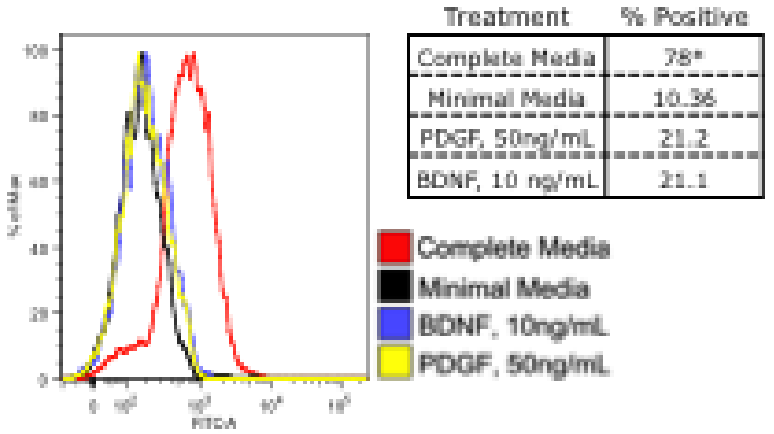

\section{BrdU Proliferation Assay}

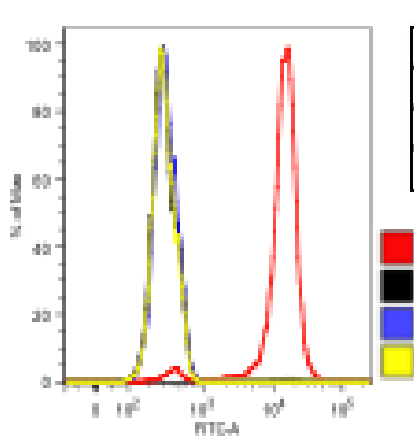

Treatment Rositive $94.95 \pm .2$ Minimal Madia $1.58^{*} \pm .7$ PDGF, $50 \mathrm{ng} / \mathrm{mL}, 10^{*}=005$ BDNF, $10 \mathrm{ne} / \mathrm{mL}$. OS $2 * .008$

Complete Medits

Minimal Meda

BDNF, 10nglmL. POCF, songimt

Figure 2: PDGF-AA and BDNF do not influence mNPC proliferation. An mNPC growth curve (A), a Ki67 flow cytometry assay (B), and a BrdU flow cytometry assay (C) demonstrate that PDGF-AA and BDNF do not stimulate NPC growth as measured after 4 days of treatment. All data is presented as compared to a complete media positive control as well as a minimal media control (a media non-conducive to cell proliferation). Data is quantified as the number of cells that stain positive for the dye over the total number of nuclei counted. *significance, $p<.05$. 
Citation: Soltys J, Perrone C, Knight J, Mao-Draayer Y (2011) PDGF-AA and BDNF Promote Neural Stem Cell Differentiation. J Neurol Neurophysiol S4. doi:10.4172/2155-9562.S4-002

Page 3 of 7
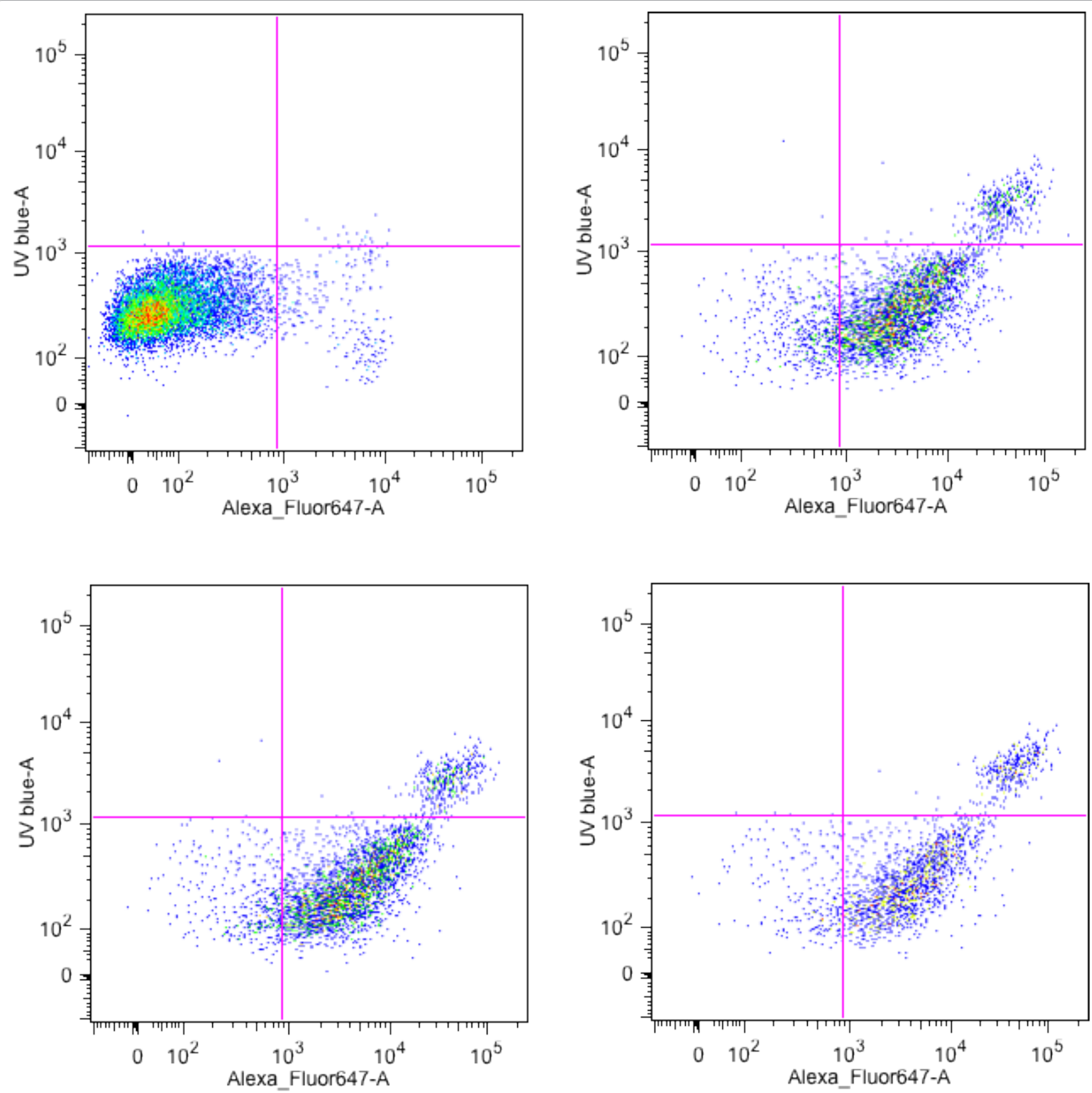

Figure 3: PDGF-AA and BDNF do not protect NPCs from undergoing apoptosis. NPCs were exposed to harsh, growth factor deprived media which induces cell death in the presence and absence of PDGF-AA and BDNF to assess the neuroprotective potential of these factors. A UVIAnnexin flow cytometry assay was used to label cells that are dead and dying. Scatterplots are presented above and the number of live healthy cells is quantified in the bottom left corner as the number of cells that do not stain for either UV or Annexin over the total number of nuclei counted. Complete media (EGF and FGF was added to the minimal media, upper left), minimal media (without EGF and FGF, upper right), BDNF in minimal media (lower left), PDGF-AA in minimal media (lower right).

Cell viability and apoptosis: A UV/annexin flow cytometry stain assessed the percentage of dead and dying cells, as described previously [22]. In brief, UV live/dead dye (Invitrogen) permeates into and reacts throughout the volume of cells which have lost their membrane integrity, thus labeling necrotic cells. Annexin-alexa fluor 647 (Invitrogen) labels phospholipid phosphatidylserine residues on the exterior surface of apoptotic cells (translocation of PS is one of the earliest indicators of apoptosis). These antibodies were added to cells after four days of treatment in culture, and analyzed using flow cytometry. All flow data is presented as a scatterplot demonstrating stained cells, and is quantified in the lower left corner of each plot as the number of cells that are not positive for either stain (ie, are double negatively labeled) over the total number of cells counted. 
A.

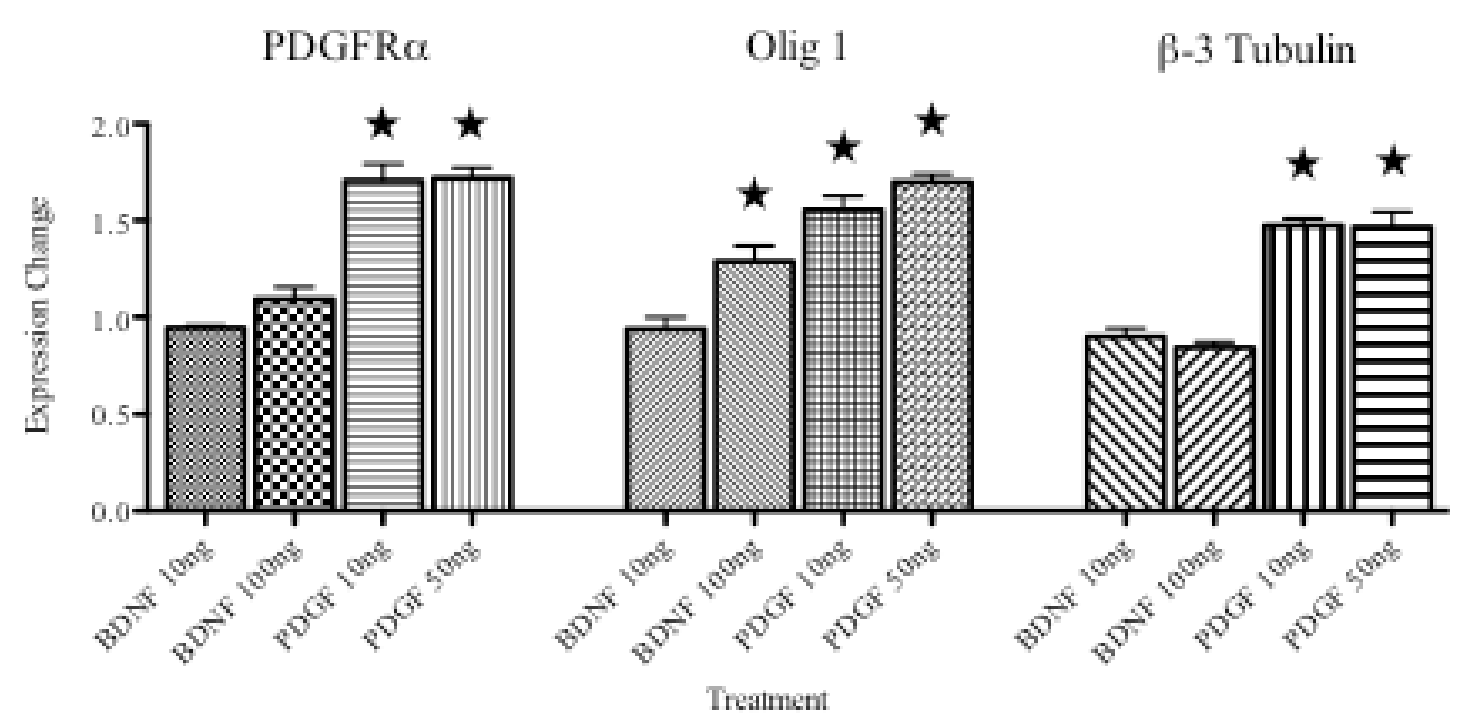

B.

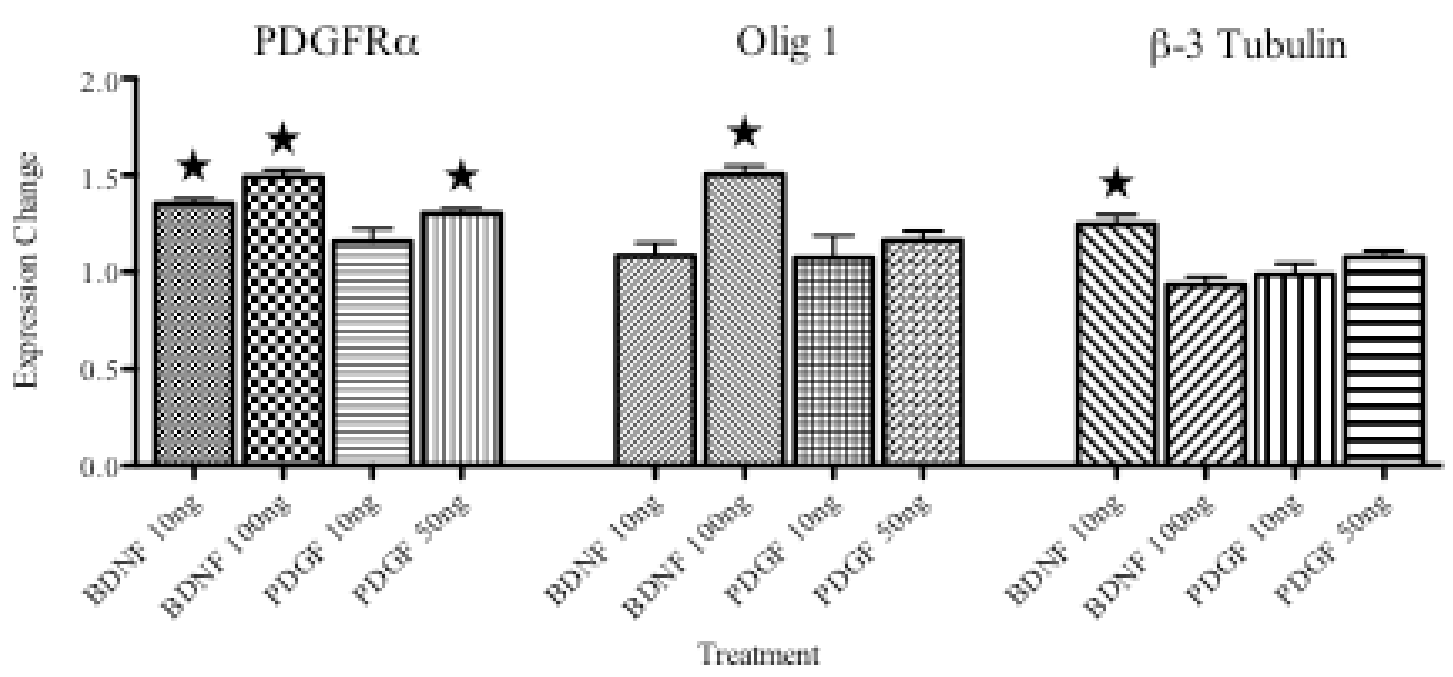

Figure 4: PDGF-AA and BDNF influence NPC differentiation. The ability for PDGF-AA and BDNF to induce NPC differentiation was assessed after 3 days (A) and 4 days (B) of treatment by RT-qPCR against PDGFRa and Olig 1 (oligo-lineage) and B3-Tub (neuronal lineage) markers. All data is presented as normalized mRNA levels against the endogenous control b-actin. *significance, $p<.05$.

RT-qPCR: Total RNA was isolated from PDGF-AA and BDNF treated cells (3-day and 4-day treatments) and minimal media controls using RNeasy Mini Kit (Qiagen) via manufacturer protocol. RNA levels were quantified by Nanodrop ${ }^{T M}$. cDNA was constructed using Superscript III ${ }^{\mathrm{TM}}$ First-Strand Synthesis Supermix for qRT-PCR (Invitrogen). mRNA expression analysis was completed using Applied Biosystems 7500 Fast Software real time-quantitative polymerase chain reaction (RT-qPCR). Amplification consisted of 50 cycles $\left(95^{\circ} \mathrm{C}\right.$ for 15 seconds and $60^{\circ} \mathrm{C}$ for 1 minute) with approximately $15 \mathrm{ng} / \mu \mathrm{L} \mathrm{cDNA}$, specific primer pairs for $\beta$-tubulin III ( $\beta 3$-tub; neuronal marker), PDGFR- $\alpha$ and Olig1 (oligodendrocyte markers); and TaqMan Master Mix (all Applied Biosystems). Transcripts were normalized against the endogenous control marker $\beta$-Actin and subsequently presented as fold differences relative to lineage marker and treatment condition. The $2^{-\Delta \Delta C t}$ method was used to calculate the relative expression of genes. Each primer was run in triplicate.

Immunohistochemistry: Cells were stained for differentiation markers and for nestin as confirmation of neural stem cell phenotype. Cells were plated on coated (as described above) coverslips in 24-well plates. After a one week treatment period, being re-treated at 4 days, coverslips were fixed in Zamboni's fixative (4\% paraformaldehyde; $15 \%$ picric acid). Cells were incubated in block (10\% horse serum, $.3 \%$ Triton) in the following antibodies at $4^{\circ} \mathrm{C}$ : $\beta$-tubulin III (Sigma; $\left.1: 200\right)$, GFAP (DAKO; 1:250), Nestin (Novus Biologicals; 1:250), PDGFRa (Santa Cruz; 1:50), O4 (COMPANY). Following 2 washes, anti-chicken 
Cy2 and anti-rabbit Cy3 (both Jackson Immunoresearch) were applied as secondary antibodies for their respective primaries for 1 hour at $4^{\circ} \mathrm{C}$. All data is shown as a representative coverslip image with quantification as the total number of positively-stained cells over the total number of nuclei counted.

\section{Statistical analysis}

One-way ANOVA was performed to determine statistical significance of the data, followed by Tukey's post-test to compare individual samples. Analyses were done using Prism 5 (Graph Pad) software. All experiments were replicated for $n \geq 3$ biological replicates.

\section{Results}

\section{PDGF-AA and BDNF do not stimulate NPC proliferation}

We investigated the influence of BDNF and PDGF-AA on mNPC proliferation in three distinct assays: quantifying absolute cell number on a growth curve (Figure 1A), a Ki67 proliferation assay (Figure 1B), and a BrdU proliferation assay (Figure 1C). Each assay demonstrates that BDNF and PDGF-AA do not stimulate NPC proliferation.

\section{PDGF-AA and BDNF do not rescue dying cells from apoptosis}

As we saw no influence and proliferation, we asked the question if PDGF-AA and BDNF are able to protect NPCs in harsh conditions that normally induce cell death. We utilized a UV/Annexin flow cytometry assay to determine the percentage of cells that do not stain positive for either UV live/dead die (labeling necrotic cells) and Annexin (labeling dying cells; Figure 2) when NPCs are exposed to growth-factor deprived media, media that will typically induce apoptosis. Neither PDGF-AA nor BDNF exert a protective effect on NPCs.

\section{PDGF-AA and BDNF influence NPC differentiation in a time-dependent manner}

As neurotrophic factors have a variety of roles in promoting differentiation, we investigated how PDGF-AA and BDNF induce NPC differentiation over time. We analyzed mRNA level expression for neuronal and oligodendrocyte-lineage markers after 3 days and 4 days of differentiation (Figures $3 \mathrm{~A}$ and $3 \mathrm{~B}$, respectively). Our findings suggest that PDGF initially stimulates differentiation non-specifically as there is global upregulation of the oligodendrocyte markers PDGFR $\alpha$ and Olig1, as well as the neuronal marker $\beta 3$-Tub. These increases were transient as 24 hours later PDGFRa was the only factor that remained upregulated with high dose PDGF-AA treatment. BDNF also nonspecifically induced mRNA expression changes over time. At 3 days, only Olig 1 expression had increased while at day 4 PDGFRa, Olig1 and 3-Tub were all increased.

As PDGF-AA has been used to induce OPC differentiation, we further examined these transient and time dependent changes by quantifying the protein expression of PDGFRa, $\beta 3$-Tub, and the astrocyte marker GFAP after 1 week of differentiation in the presence of PDGF-AA (Figure 5). At this time point most cells are committed to differentiation, indicated by the very small percentage of cells that only stain positive for the NPC marker nestin (Figure $5 \mathrm{I}-\mathrm{K}$ ). $10 \mathrm{ng} / \mathrm{ml}$ PDGF-AA treatment increases the percentage of cells that stain positive for PDGFR $\alpha$, without changes in the expression of $\beta 3$-Tub and GFAP as compared to MM control. As PDGFRa is a more immature marker for differentiation, we further investigated this finding by staining for $\mathrm{O} 4$, a marker for more mature oligodendrocytes. After 1 week in culture, no cells stained positive for $\mathrm{O} 4$ (Figure $5 \mathrm{~N}$ ).
To verify that these findings represent differentiation from a pure neural stem cell population, we stained our cell population with the NPC marker nestin prior to differentiation media exposure. Our NPC population is pure, as every cell (detected by the nuclear stain Hoechst) stains positive for nestin (Figure $5 \mathrm{M}$ ).

\section{Discussion}

The key finding of this study is that PDGF-AA stimulates NPC differentiation into the oligodendrocyte lineage (Figure 4) and we provide further confirmation that BDNF and PDGF-AA are not toxic to NPCs (Figures 1 and 2).

Our finding that BDNF is capable of stimulating NPC differentiation is consistent with previous work done by Silva et al. [23] in which they assessed the differentiation potential of neurospheres grown in culture when exposed to BDNF. They found increased differentiation of NPCs into the neuronal lineage after 4 days in vitro, which can be manipulated by the presence of other extracellular factors. We confirm this finding via RT-qPCR and further suggest that NPCs are capable of differentiating into the oligodendrocyte lineage as we observed elevated expression of PDGFR $\alpha$ and Olig1 in response to BDNF treatment (Figure 3). Notably, there appears to be time-dependent expression of mRNA for the various neuronal lineages. Thus, BDNF appears to prime the NSC for differentiation by upregulating the neuronal and oligodendrocyte lineage pathways, but ultimately other factors present in the microenvironment may be the critical factors in driving NSC fate.

NSC differentiation into the oligodendrocyte lineage was seen after one week of PDGF-AA treatment (Figure 4). While PDGF-AA is a potent mitogen for OPCs [20,24], the definitive role it plays in inducing NPCs into OPCs is not completely understood. Other studies performed with embryonic stem cells suggest that it is capable of inducing both neuronal and oligo-lineage differentiation [19,25]. Here we use a postnatal murine line to investigate this further as there are known differences between embryonic and postnatal stem cells. We confirm the capacity for PDGF-AA to induce NPC differentiation into the oligodendrocyte lineage in post-natal NPCs. Importantly, PDGF responsive cells are present in high numbers in MS lesions and likely play a critical role in remyelinating damaged lesions. Chronically, however, this population may be depleted [26]. Taken together, our findings suggest that PDGF-AA is capable of stimulating NPCs to refill this pool. Future investigations that quantify the level of PDGF-AA expression over time in inflammatory lesions, as well the NPC response to therapeutic administrations of PDGF-AA will likely prove to be fruitful in elucidating how NPCs respond to the need for remyelination as well further explain why the NPC response ultimately fails.

Neurotrophins and neurotrophic cytokines that regulate neuronal and oligodendrocyte development are deficient in MS [27,28]. The benefits of therapeutic administration of these factors is highlighted by in vivo studies that demonstrate the neuroprotective properties of BDNF and PDGF-AA when applied acutely to inflammatory lesions [15-17,29]. Adapting these approaches for more chronic protection demands knowledge of which cells these factors influence so that they can be stimulated at the appropriate time and anatomical position. Thus, our in vitro approach that investigates the molecular response of NPCs to these factors is critical to identifying the environment needed for remyelination. By coupling this basic knowledge with future investigations that quantify the expression level changes of neurotrophic factors over time in MS pathogenesis, we can begin to 


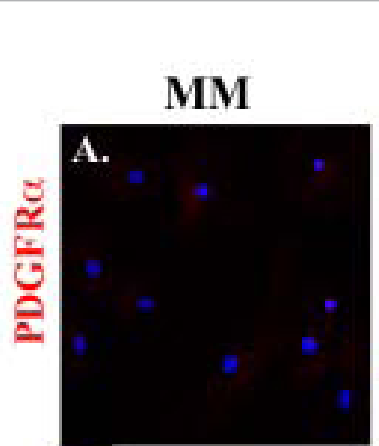

\section{PDGF Treatment}

\section{$10 \mathrm{ng} / \mathrm{ml}$}
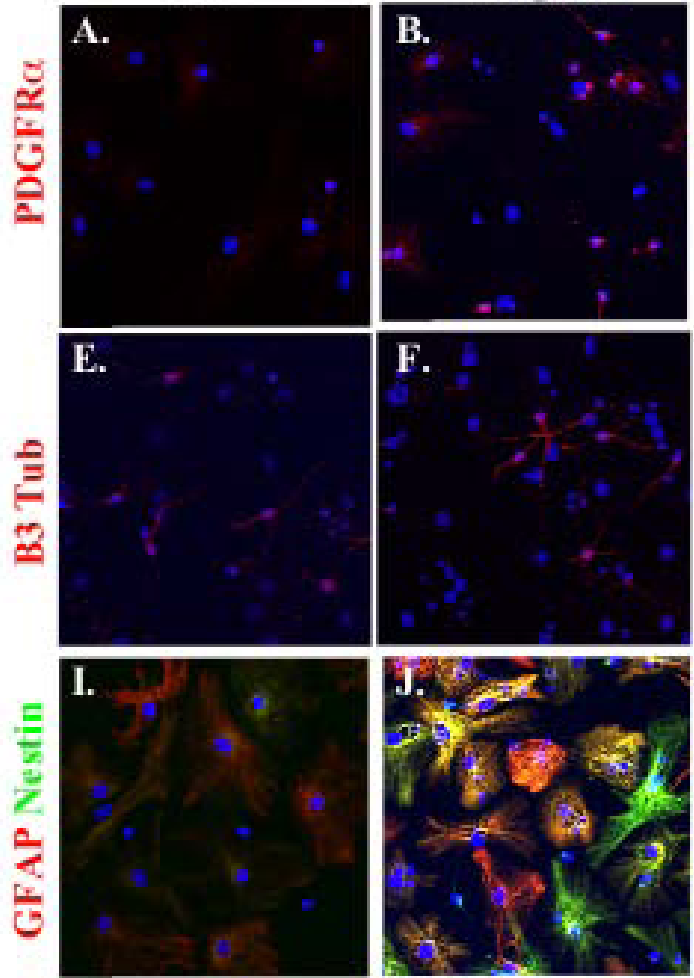
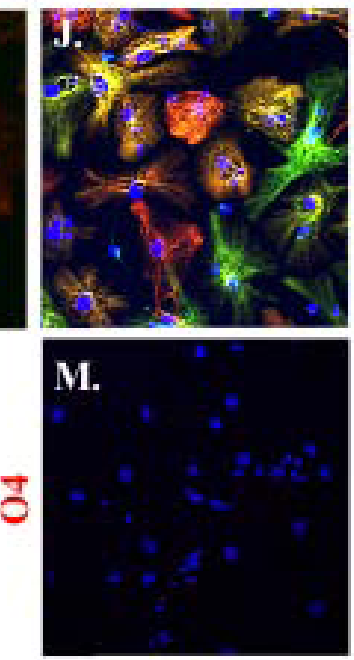

$50 \mathrm{ng} / \mathrm{ml}$
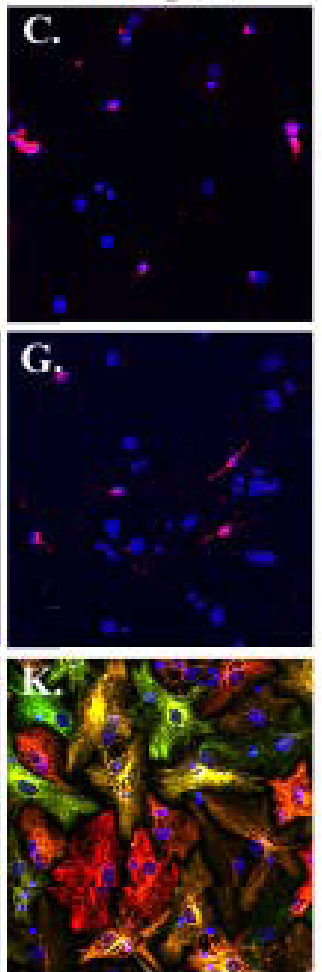

Confocal

Quantification

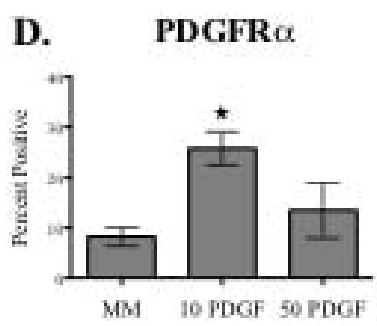

H. B3 Tub

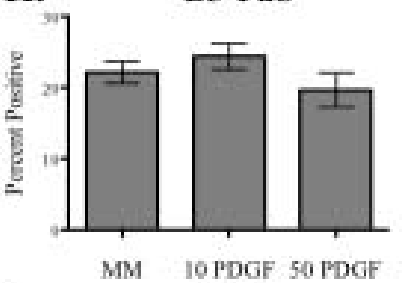

L.

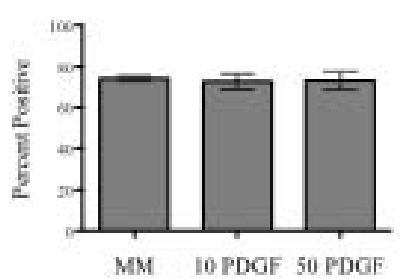

Figure 5: PDGF-AA treatment promotes oligo-lineage differentiation. Immunohistochemistry against PDGFRa (oligo-lineage, A-D), B3-Tub (neuronal lineage, E-H), GFAP (astrocyte lineage, I-L), and O4 (Mature oligo lineage, M) was used to determine how PDGF-AA induces differentiation after 1 week of treatment. All images represent a representative confocal field of view, and quantification $(D, H, I)$ is presented as the total number of positively stained cells over the total number of cells per field, counted by the nuclear marker Hoechst. At one week, nearly all cells are differentiated, as determined by the absence of the neural stem cell marker nestin (I, J, K). * significance, $\mathrm{p}<.05$

design neurotrophic factor therapies that directly combat disease pathology by maintaining a population of cells capable of remyelination.

In conclusion, our findings demonstrate that BDNF and PDGF-AA do not stimulate NPC proliferation or induce apoptosis, but are capable of inducing NPC differentiation into the oligodendrocyte lineage. The differentiation capabilities are time dependent and may depend on other extracellular factors. These findings highlight the prospects of stem cell therapies to promote remyelination of MS lesions, and demand future studies to quantify the expression levels of neurotrophic factors such as BDNF and PDGF-AA over the MS disease course in order to design replacement therapies that can promote more efficient remyelination.

\section{Acknowledgements}

We would like to thank the Center of Biomedical Research Excellence (COBRE) at the University of Vermont for their continued support and equipment access. Confocal microscopy was done at the University of Vermont Microscopy
Imaging Center (MIC), and the project described was supported by award number 1S10 RR019246-01 from the National Center for Research Resources, which funded the purchase of the confocal microscope in the MIC.

\section{References}

1. Patani R, Balaratnam M, Vora A, Reynolds R (2007) Remyelination can be extensive in multiple sclerosis despite a long disease course. Neuropathol Appl Neurobiol 33: 277-287.

2. Prineas JW, Kwon EE, Cho ES, Sharer LR, Barnett MH, et al. (2001) Immunopathology of secondary-progressive multiple sclerosis. Ann Neurol 50: 646-657.

3. Franklin RJ (2002) Why does remyelination fail in multiple sclerosis? Nat Rev Neurosci 3: 705-714

4. Kuhlmann T, Miron V, Cui Q, Wegner C, Antel J, et al. (2008) Differentiation block of oligodendroglial progenitor cells as a cause for remyelination failure in chronic multiple sclerosis. Brain 131: 1749-1758.

5. Martino G, Pluchino S (2006) The therapeutic potential of neural stem cells. Nat Rev Neurosci 7: 395-406. 
Citation: Soltys J, Perrone C, Knight J, Mao-Draayer Y (2011) PDGF-AA and BDNF Promote Neural Stem Cell Differentiation. J Neurol Neurophysiol S4. doi:10.4172/2155-9562.S4-002

Page 7 of 7

6. Bibel M, Barde YA (2000) Neurotrophins: key regulators of cell fate and cell shape in the vertebrate nervous system. Genes Dev 14: 2919-2937.

7. Glebova NO, Ginty DD (2005) Growth and survival signals controlling sympathetic nervous system development. Annu Rev Neurosci 28: 191-222.

8. Lindquist S, Hassinger S, Lindquist JA, Sailer M (2011) The balance of proinflammatory and trophic factors in multiple sclerosis patients: effects of acute relapse and immunomodulatory treatment. Mult Scler 17: 851-866.

9. Rudick RA, Ransohoff RM, Lee JC, Peppler R, Yu M, et al. (1998) In vivo effects of interferon beta-1a on immunosuppressive cytokines in multiple sclerosis. Neurology 50: 1294-1300.

10. Diemel LT, Jackson SJ, Cuzner ML (2003) Role for TGF-beta1, FGF-2 and PDGF-AA in a myelination of CNS aggregate cultures enriched with macrophages. J Neurosci Res 74: 858-867.

11. Harirchian $\mathrm{MH}$, Tekieh $\mathrm{AH}$, Modabbernia A, Aghamollaii $\mathrm{V}$, Tafakhori A, et al (2011) Serum and CSF PDGF-AA and FGF-2 in relapsing-remitting multiple sclerosis: a case-control study. Eur J Neurol [Epub ahead of print]

12. Maeda Y, Solanky M, Menonna J, Chapin J, Li W, et al. (2001) Platelet-derived growth factor-alpha receptor-positive oligodendroglia are frequent in multiple sclerosis lesions. Ann Neurol 49: 776-785.

13. Wilson HC, Scolding NJ, Raine CS (2006) Co-expression of PDGF alpha receptor and NG2 by oligodendrocyte precursors in human CNS and multiple sclerosis lesions. J Neuroimmunol 176: 162-173.

14. Nagahara AH, Tuszynski MH (2011) Potential therapeutic uses of BDNF in neurological and psychiatric disorders. Nat Rev Drug Discov 10: 209-219.

15. Makar TK, Bever CT, Singh IS, Royal W, Sahu SN, et al. (2009) Brain-derived neurotrophic factor gene delivery in an animal model of multiple sclerosis using bone marrow stem cells as a vehicle. J Neuroimmunol 210: 40-51.

16. McTigue DM, Horner PJ, Stokes BT, Gage FH (1998) Neurotrophin-3 and brain-derived neurotrophic factor induce oligodendrocyte proliferation and myelination of regenerating axons in the contused adult rat spinal cord. $J$ Neurosci 18: 5354-5365.

17. Vana AC, Flint NC, Harwood NE, Le TQ, Fruttiger M, et al. (2007) Plateletderived growth factor promotes repair of chronically demyelinated white matter. J Neuropathol Exp Neurol 66: 975-988.

18. Erlandsson A, Enarsson M, Forsberg-Nilsson K (2001) Immature neurons from CNS stem cells proliferate in response to platelet-derived growth factor. $J$ Neurosci 21: 3483-3491.

19. Hu JG, Fu SL, Wang YX, Li Y, Jiang XY, et al. (2008) Platelet-derived growth factor-AA mediates oligodendrocyte lineage differentiation through activation of extracellular signal-regulated kinase signaling pathway. Neuroscience 151 138-147.

20. Baron W, Shattil SJ, Ffrench-Constant C (2002) The oligodendrocyte precurso mitogen PDGF stimulates proliferation by activation of alpha(v)beta3 integrins. EMBO J 21: 1957-1966.

21. Snethen H, Love S, Scolding N (2008) Disease-responsive neural precursor cells are present in multiple sclerosis lesions. Regen Med 3: 835-847.

22. Knight JC, Scharf EL, Mao-Draayer Y (2010) Fas activation increases neura progenitor cell survival. J Neurosci Res 88: 746-757.

23. Silva A, Pereira J, Oliveira CR, Relvas JB, Rego AC (2009) BDNF and extracellular matrix regulate differentiation of mice neurosphere-derived cells into a GABAergic neuronal phenotype. J Neurosci Res 87: 1986-1996.

24. Baron W, Metz B, Bansal R, Hoekstra D, de Vries H (2000) PDGF and FGF2 signaling in oligodendrocyte progenitor cells: regulation of proliferation and differentiation by multiple intracellular signaling pathways. Mol Cell Neurosci 15: 314-329.

25. Demoulin JB, Enarsson M, Larsson J, Essaghir A, Heldin CH, et al. (2006) The gene expression profile of PDGF-treated neural stem cells corresponds to partially differentiated neurons and glia. Growth Factors 24: 184-196.

26. Miron VE, Kuhlmann T, Antel JP (2011) Cells of the oligodendroglial lineage, myelination, and remyelination. Biochim Biophys Acta 1812: 184-193.

27. Caggiula M, Batocchi AP, Frisullo G, Angelucci F, Patanella AK, et al. (2005) Neurotrophic factors and clinical recovery in relapsing-remitting multiple sclerosis. Scand J Immunol 62: 176-182.

28. Urshansky N, Mausner-Fainberg K, Auriel E, Regev K, Farhum F, (2010) Dysregulated neurotrophin mRNA production by immune cells of patients with relapsing remitting multiple sclerosis. J Neurol Sci 295: 31-37.

29. Linker R, Gold R, Luhder F (2009) Function of neurotrophic factors beyond the nervous system: inflammation and autoimmune demyelination. Crit Rev Immunol 29: 43-68.

This article was originally published in a special issue, Neurorehabilitation \& Neural Repair handled by Editor(s). Dr. Hsinlin Thomas Cheng, University of Michigan, USA 